\title{
Fractional-Order Terminal Sliding-Mode Control for Buck DC/DC Converter
}

\author{
Ningning Yang, ${ }^{1,2}$ Chaojun $\mathrm{Wu}^{3}{ }^{3}$ Rong Jia, ${ }^{1,2}$ and Chongxin Liu ${ }^{4}$ \\ ${ }^{1}$ State Key Laboratory Base of Eco-Hydraulic Engineering in Arid Area, Xi'an University of Technology, Xian 710048, China \\ ${ }^{2}$ Institute of Water Resources and Hydro-Electric Engineering, Xian University of Technology, Xian 710048, China \\ ${ }^{3}$ College of Electronics and Information, Xi'an Polytechnic University, Xian 710048, China \\ ${ }^{4}$ School of Electrical Engineering, Xian Jiaotong University, Xian 710049, China \\ Correspondence should be addressed to Chaojun Wu; chaojun.wu@stu.xjtu.edu.cn
}

Received 11 March 2016; Revised 14 June 2016; Accepted 3 July 2016

Academic Editor: Anna Pandolfi

Copyright ( 2016 Ningning Yang et al. This is an open access article distributed under the Creative Commons Attribution License, which permits unrestricted use, distribution, and reproduction in any medium, provided the original work is properly cited.

In recent years, the combination of fractional calculus (FC) and sliding-mode control (SMC) has been gaining more and more interests due to fusion characteristics of SMC and FC. This paper presents the fractional-order terminal sliding-mode control (FTSMC) which has a new fractional-order sliding surface and assures the finite time convergence of the output voltage error to the equilibrium point during the load changes. TSMC is a special case of FTSMC. Through mathematical analysis, the system can reach the sliding-mode surface in finite time. The theoretical considerations have been verified by numerical simulations. And a Buck DC/DC converter application is presented and compared to illustrate the effectiveness of the proposed method. It is shown that the novel fractional terminal sliding-mode control exhibits considerable improvement in terms of a faster output voltage response during load changes.

\section{Introduction}

The concept of fractional calculus as an extension of ordinary calculus can stretch back to 1695 . In the letter to L'Hospital, Leibniz proposed the possibility of generalizing the operation of half-order derivative [1]. Though fractional calculus has a long history, only in recent years have the applications of fractional calculus to physics and engineering become an important aspect of modern technology. In comparison with the classical elementary calculus, the main advantage of fractional calculus is that it can provide an elegant description for the memory and hereditary properties of various real objects. The list of the applied fields of fractional calculus has been ever growing and includes the electrode-electrolyte polarization, viscoelastic fluids, chaotic systems, and power converters [2-6]. Most striking of all, the fractional differentials and integrals are applied to the control theory, when the controller or the controlled system is described by fractional calculus [7-11]. In 1996, Oustaloup et al. developed the first fractional-order controller which is the so-called CRONE [12]. Then some fractional-order control strategies are proposed one after another, such as fractionalorder PID controllers [13], fractional-order sliding-mode controllers [14-16], fractional-order optimal controllers [17], and fractional-order adaptive controllers $[18,19]$.

DC/DC power converters, which work in switch mode, are applied in a wide variety of applications, including DC motor drives, active filters, computers, power supplies, and medical instrumentation. The Buck converter is one of the simplest but most useful power converters that can convert a DC input to a DC output at a lower voltage. There are some other basic DC/DC converters, namely, the Boost, Buck-Boost, Cuk, Zeta, and Sepic. Each of these converters consists of the passive power switch, the active power switch, and the storage elements. The main objective of most closed-loop feedback controlled DC/DC converters is to ensure that the converter operates with fast dynamical response, small steady-state output error, and low overshoot, while maintaining high efficiency and low noise emission in terms of rejection of input voltage changes, parameter 
uncertainties, and load variations [20]. The designing of high performance control strategy is always a challenge, because DC/DC converters are inherently time-varying variablestructure nonlinear systems. Nonlinear control strategies [21-23] are better candidates in DC/DC converters than other linear ones. Among the nonlinear control strategies, the sliding-mode control (SMC) has been well known due to its fast dynamic response, robustness to disturbances, guaranteed stability, parameter variations, and simplicity in implementation. Moreover, compared with other nonlinear control strategies, the SMC method is easy to implement and has a high degree of flexibility in the designing process.

The sliding-mode control is an effective robust control strategy with the feature of switching the control law to force the state trajectories of the system from the initial states onto some predefined sliding surface which exhibit desired dynamics. When in the sliding mode, the closedloop response becomes totally insensitive to both internal parameter uncertainties and external disturbances. Compared with the SMC, the terminal sliding-mode control (TSMC) has some superior properties, such as the state of the system which converges in finite time and the higher steady-state tracking accuracy. Near the equilibrium point the rate of the convergence is being speeded up, so the TSMC is more suitable for high precision control. In recent years, the combination of fractional-order control (FOC) and slidingmode control (SMC) has been gaining more and more interests from the systems control community. An introductory work on the application in fractional-order sliding mode is reported in [24]; the double integrator is introduced as the plant under control. A fuzzy fractional-order sliding-mode controller is adopted to control nonlinear systems in [25]. In [26], fractional-order sliding-mode control strategies for power electronic Buck converters are presented, where pulsewidth modulation (PWM) sets the basis for the regulation of switched mode converters. In [27], authors collect different methods to apply FOC in SMC through the use of fractionalorder surfaces which are fractional-order PID or fractionalorder PI and propose a direct Boolean control strategy in order to avoid using PWM.

In this paper, we focus on the introduction of the FOC in TSMC for switching systems. The fractional-order terminal sliding-mode control (FTSMC) method has been adopted for controlling the Buck converter. The idea of such method is used to design a novel nonlinear sliding surface function which is a fusion of characteristics of TSMC and FOC. Based on the Lyapunov stability theory, a fractional-order sliding-mode control law is derived to assure finite time convergence of the output voltage error to the equilibrium point. Through mathematical analysis, we obtain the finite time $t_{s}^{\text {FTSMC }}$ of FTSMC. It is shown that TSMC is a special case of FTSMC. The theoretical considerations have been verified by numerical simulations and a Buck converter application is given to show the superiority of the proposed strategy. The fractional terminal sliding-mode control exhibits considerable improvement in terms of a faster output voltage response during load changes. FTSMC can overcome the influence which disturbances bring about to control system and guarantee that DC/DC converter keeps good dynamic and steady performances. The output can follow the given well, and the disturbances almost do not affect the output.

The rest of this paper is organized as follows. In Section 2, some basic concept and definitions of the fractional calculus are introduced. Then the basic principle of the Buck converter in CCM is introduced. In Section 3, the integer-order TSMC is briefly reviewed. In Section 4, FTSMC is described for the Buck converter. In Section 5, numerical simulation results are presented to verify the theoretical considerations and an application to Buck converter is given to show the superiority of the proposed strategy. Finally, some concluding remarks of this paper are drawn in Section 6.

\section{Preliminaries}

In this section, firstly, some basic concept and definitions of fractional calculus are introduced. Afterwards, a brief introduction to the basic principle of the Buck converter in CCM is presented.

2.1. The Basis of Fractional Calculus. In fractional calculus, the operator ${ }_{a} D_{t}^{\alpha}$ is the differintegral operator which takes both integrals and derivatives in one single expression. It denotes generalization of integrals and derivatives to arbitrary order and can be defined as follows:

$$
{ }_{a} D_{t}^{\alpha}= \begin{cases}\frac{d^{\alpha}}{d t^{\alpha}}, & \operatorname{Re}[\alpha]>0 \\ 1, & \operatorname{Re}[\alpha]=0 \\ \int_{a}^{t}(d \tau)^{-\alpha}, & \operatorname{Re}[\alpha]<0,\end{cases}
$$

where $\alpha \in R$ can be an arbitrary real number, which denotes the order of the operation, and $a$ and $t$ are the lower and upper limits.

Definition 1. In the fractional calculus, the Gamma function proposed by Euler is defined by the integral

$$
\Gamma(z)=\int_{0}^{\infty} e^{-t} t^{z-1} d t
$$

which satisfies $\operatorname{Re}(z)>0$, and converges in the right half of the complex plane.

Several reputed definitions for fractional derivatives are put forward including Riemann-Liouville definition, Grunwald-Letnikov definition, Caputo definition, Weyl definition, and Marchaud definition. Among them, being the best-known one, Riemann-Liouville definition is precisely studied.

Definition 2 (see [7]). Riemann-Liouville definition of the $\alpha$ th-order fractional derivative operator ${ }_{0} D_{t}^{\alpha}$ is given by

$$
{ }_{0} D_{t}^{\alpha} f(t)=\frac{1}{\Gamma(1-\alpha)} \frac{d}{d t} \int_{0}^{t}(t-\tau)^{-\alpha} f(\tau) d \tau \text {, }
$$

where $0<\alpha<1$. 


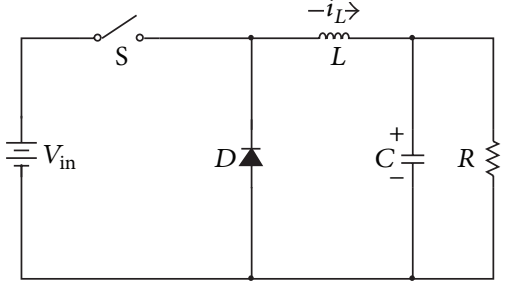

(a)

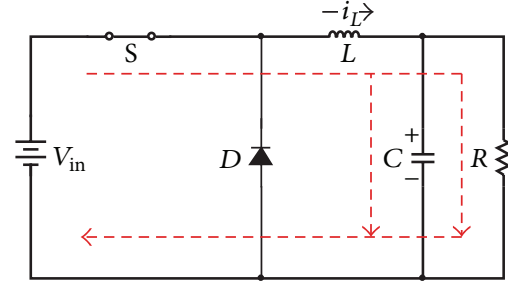

(b)

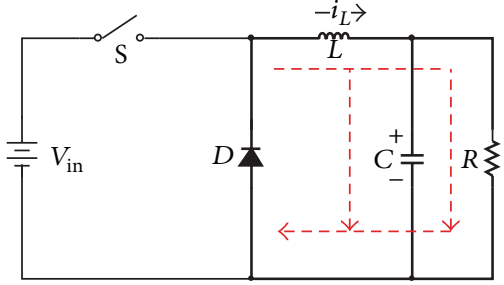

(c)

FIGURE 1: (a) Buck converter; (b) the switch $\mathrm{S}$ is turned on; (c) the switch $\mathrm{S}$ is turned off.

Definition 3 (see [7]). Riemann-Liouville definition of the $\alpha$ th-order fractional derivative operator ${ }_{0} D_{t}^{-\alpha}$ is defined by

$$
{ }_{0} D_{t}^{-\alpha} f(t)=\frac{1}{\Gamma(\alpha)} \int_{0}^{t}(t-\tau)^{\alpha-1} f(\tau) d \tau
$$

According to this definition, the derivative of a time function with $f(t)=t^{\beta}, \beta>-1$, is evaluated as

$$
{ }_{0} D_{t}^{\alpha} t^{\beta}=\frac{\Gamma(\alpha+1)}{\Gamma(\alpha-\beta+1)} t^{\beta-\alpha} .
$$

Definition 4 (see [7]). If the fractional derivative ${ }_{0} D_{t}^{\alpha} f(t)(k-1<\alpha<k)$ and a function $f(t)$ are integrable, then

$$
\begin{aligned}
& { }_{a} D_{t}^{-\alpha}\left({ }_{0} D_{t}^{-\alpha} f(t)\right) \\
& \quad=f(t)-\sum_{j=1}^{k}\left[{ }_{0} D_{t}^{\alpha-j} f(t)\right]_{t=a} \frac{(t-a)^{\alpha-j}}{\Gamma(\mu-j+1)} .
\end{aligned}
$$

2.2. Brief Introduction of the Buck Converter in CCM. The Buck converter, sometimes called a step-down power stage, is a popular nonisolated power stage topology. A simplified schematic of the Buck converter is shown in Figure 1(a).

The basic operation of the Buck converter can be regarded as piecewise switched dynamical systems, involving toggling among a set of linear or nonlinear circuit topologies. In continuous conduction mode (CCM), there are two switching modes. (1) When the switch (labeled as S in Figure 1(b)) is turned on, the inductor current passes through the switch, and the diode is reverse-biased with the inductor current $i_{L}$ ramping up. (2) When the switch (labeled as S in Figure 1(c)) is turned off, the inductor maintains current flow in the same direction so that the diode is forward-biased. And this causes the inductor current to ramp down. The process repeats cyclically. For the operation described above, we have two state equations as follows.

When $\mathrm{S}$ is turned on,

$$
\left[\begin{array}{l}
\frac{d i_{L}(t)}{d t} \\
\frac{d v_{o}(t)}{d t}
\end{array}\right]=\left[\begin{array}{cc}
0 & -\frac{1}{L} \\
\frac{1}{C} & -\frac{1}{C R}
\end{array}\right]\left[\begin{array}{l}
i_{L}(t) \\
v_{o}(t)
\end{array}\right]+\left[\begin{array}{c}
\frac{1}{L} \\
0
\end{array}\right] v_{\text {in }}(t) .
$$

When $\mathrm{S}$ is turned off,

$$
\left[\begin{array}{l}
\frac{d i_{L}(t)}{d t} \\
\frac{d v_{o}(t)}{d t}
\end{array}\right]=\left[\begin{array}{cc}
0 & -\frac{1}{L} \\
-\frac{1}{C} & -\frac{1}{C R}
\end{array}\right]\left[\begin{array}{l}
i_{L}(t) \\
v_{o}(t)
\end{array}\right]+\left[\begin{array}{l}
0 \\
0
\end{array}\right] v_{\text {in }}(t) .
$$

Combining (7) and (8) gives

$$
\left[\begin{array}{l}
\frac{d i_{L}(t)}{d t} \\
\frac{d v_{o}(t)}{d t}
\end{array}\right]=\left[\begin{array}{cc}
0 & -\frac{1}{L} \\
\frac{1}{C} & -\frac{1}{C R}
\end{array}\right]\left[\begin{array}{l}
i_{L}(t) \\
v_{o}(t)
\end{array}\right]+\left[\begin{array}{l}
\frac{1}{L} \\
0
\end{array}\right] u v_{\text {in }}(t),
$$

where $u$ is the control input. The switch is in the ON state for $u=1$ and in the OFF state for $u=0$.

Define the output voltage error $x_{1}$ as follows:

$$
x_{1}=v_{o}-V_{\text {ref }} \text {, }
$$

where $V_{\text {ref }}$ is the reference value of the output voltage. By taking the time derivative of (10), $x_{2}$ which is the rate of change of voltage error can be expressed as

$$
x_{2}=\dot{x}_{1}=\dot{v}_{o} .
$$

Then the dynamics of $x_{2}$ can be given as

$$
\dot{x}_{2}=\frac{1}{L C}\left(u V_{\text {in }}-V_{\text {ref }}-x_{1}\right)-\frac{x_{2}}{R C} .
$$

So, it can be concluded that

$$
\left[\begin{array}{l}
\dot{x}_{1} \\
\dot{x}_{2}
\end{array}\right]=\left[\begin{array}{cc}
0 & 1 \\
-\frac{1}{L C} & -\frac{1}{R C}
\end{array}\right]\left[\begin{array}{l}
x_{1} \\
x_{2}
\end{array}\right]+\left[\begin{array}{c}
0 \\
u V_{\text {in }}-V_{\text {ref }}
\end{array}\right] \frac{1}{L C}
$$

\section{The Integer-Order Terminal Sliding-Mode Control for the Buck Converter}

The conventional TSM concept can be described as

$$
s=\alpha x_{1}^{\beta}+x_{2},
$$

where $\alpha$ is a designed positive constant, $\beta=q / p$, and $p$ and $q$ are both positive odd integers which satisfy the following condition: $0<q / p<1$. When the system is in the terminal sliding mode $(s=0)$, we can get its dynamics from (14):

$$
\dot{x}_{1}=-\alpha x_{1}^{\beta} \text {. }
$$


Rewriting (15) yields

$$
d t=-\frac{1}{\alpha x_{1}^{\beta}} d x
$$

Given any initial state $x_{1}(0) \neq 0$ and $x_{1}\left(t_{s}\right)=0$ which is the terminal attractor of the system. Taking integral of both sides of (16), the finite time is $t_{s}$ obtained by

$$
s=\alpha x_{1}^{\beta}+{ }_{t_{0}} D_{t}^{\mu-1} x_{2} .
$$

This means that state $x_{1}$ can converge to zero in a finite time. In other words, the term "terminal" referred to equilibrium zero and can be reached in finite time and it is stable:

$$
\begin{aligned}
\dot{s} & =\alpha \beta x_{1}^{\beta-1} \dot{x}_{1}+\dot{x}_{2} \\
& =\alpha \beta x_{1}^{\beta-1} x_{2}+\frac{1}{L C}\left(u V_{\text {in }}-V_{\text {ref }}-x_{1}\right)-\frac{x_{2}}{R C}=0 .
\end{aligned}
$$

The equivalent control can be expressed as

$$
u_{\mathrm{eq}}=\left(\frac{x_{2}}{R C}-\alpha \beta x_{1}^{\beta-1} x_{2}\right) \frac{L C}{V_{\mathrm{in}}}+\frac{\left(V_{\mathrm{ref}}+x_{1}\right)}{V_{\mathrm{in}}} .
$$

\section{The Fractional-Order Terminal Sliding-Mode Control Method for the Buck Converter}

Theorem 5. In order to introduce the so-called fractionalorder terminal sliding-mode model, a fractional-order sliding surface function can be defined as

$$
s=\alpha x_{1}^{\beta}+{ }_{t_{0}} D_{t}^{\mu-1} x_{2},
$$

where $\mu \in(0,1], \alpha$ is a designed positive constant, $\beta=q / p$, and $p$ and $q$ are both positive odd integers which satisfy the following condition: $0<q / p<1$. For Buck converter with FTSMC, if the control is designed as

$$
\begin{aligned}
u= & {\left[\frac{x_{2}}{R C}-D^{1-\mu}\left(\alpha \beta x_{1}^{\beta-1} x_{2}\right)\right] \frac{L C}{V_{\text {in }}}+\frac{\left(V_{\text {ref }}+x_{1}\right)}{V_{\text {in }}} } \\
& -K \operatorname{sign}(s)-K s,
\end{aligned}
$$

where $0<\beta<1, k>0,0<\mu<1$, then states $x_{1}$ and $x_{2}$ will converge to zero in finite time. Furthermore, the system will be in the terminal sliding mode.

Proof. Select the Lyapunov function as

$$
V=\frac{1}{2} s^{2}
$$

Taking the derivative of (22),

$$
\dot{V}=s \dot{s}=s\left(\alpha \beta x_{1}^{\beta-1} \dot{x}_{1}+D^{\mu-1} \dot{x}_{2}\right) \text {. }
$$

Substituting (12) into (23) results in

$$
\begin{aligned}
\dot{V}= & s \alpha \beta x_{1}^{\beta-1} x_{2} \\
& +s D^{\mu-1}\left[\frac{1}{L C}\left(u V_{\text {in }}-V_{\text {ref }}-x_{1}\right)-\frac{x_{2}}{R C}\right] .
\end{aligned}
$$

Substituting (21) into (24) results in

$$
\dot{V}=s(-K \operatorname{sign}(s)-K s)=-K\left(|s|+s^{2}\right) \text {. }
$$

Because the controller parameter $K$ is positive, we can find $V>0$ and $\dot{V}>0$. In other words, the controlled system satisfies the reaching condition.

When the system reaches the sliding surface $(s=0)$, it is in the terminal sliding mode. Its dynamics can be determined by the following equation:

$$
{ }_{0} D_{t_{s}}^{\mu-1} \dot{x}_{1}=-\alpha_{0} x_{1}^{\beta} \text {. }
$$

Taking the concept of fractional integral and derivative operators into account, one obtains

$$
{ }_{0} D_{t_{s}}^{1-\mu}\left({ }_{0} D_{t_{s}}^{\mu-1} \dot{x}_{1}\right)=-\alpha_{0} D_{t_{s}}^{1-\mu} x_{1}^{\beta} .
$$

From (6), we can get

$$
\begin{gathered}
\dot{x}_{1}(t)-\left[{ }_{0} D_{t}^{\mu-2} \dot{x}_{1}(t)\right]_{t=0} \frac{\left(t-t_{r}\right)^{\mu-2}}{\Gamma(\mu-1)} \\
=-\alpha_{0} D_{t_{s}}^{1-\mu} x_{1}^{\beta}(t),
\end{gathered}
$$

where $\left[{ }_{0} D_{t}^{\mu-2} \dot{x}_{1}(t)\right]_{t=0}\left(\left(t-t_{r}\right)^{\mu-2} / \Gamma(\mu-1)\right)=0$. So, it can be concluded that

$$
\dot{x}_{1}(t)=-\alpha_{0} D_{t_{s}}^{1-\mu} x_{1}^{\beta}(t) .
$$

According to Definition 3, we have

$$
D^{1-\mu} x_{1}^{\beta}(t)=\frac{\Gamma(\beta+1)}{\Gamma(\beta+\mu)} x_{1}^{\beta+\mu-1}(t) .
$$

Substituting (30) into (29) results in

$$
\dot{x}_{1}(t)=-\frac{\alpha \Gamma(\beta+1)}{\Gamma(\beta+\mu)} x_{1}^{\beta+\mu-1}(t) .
$$

Equation (31) can be rewritten as

$$
d x_{1}(t)=-\frac{\alpha \Gamma(\beta+1)}{\Gamma(\beta+\mu)} x_{1}^{\beta+\mu-1}(t) d t .
$$

Taking integral of both sides of (32),

$$
\int_{0}^{t_{s}} x_{1}^{1-\beta-\mu}(t) d x_{1}(t)=-\frac{\alpha \Gamma(\beta+1)}{\Gamma(\beta+\mu)} \int_{0}^{t_{s}} d t .
$$

The finite time $t_{s}$ is obtained by

$$
t_{s}^{\mathrm{FTSMC}}=\frac{\Gamma(\beta+\mu) x(0)^{2-\mu-\beta}}{\alpha \Gamma(\beta+1)(2-\mu-\beta)} .
$$




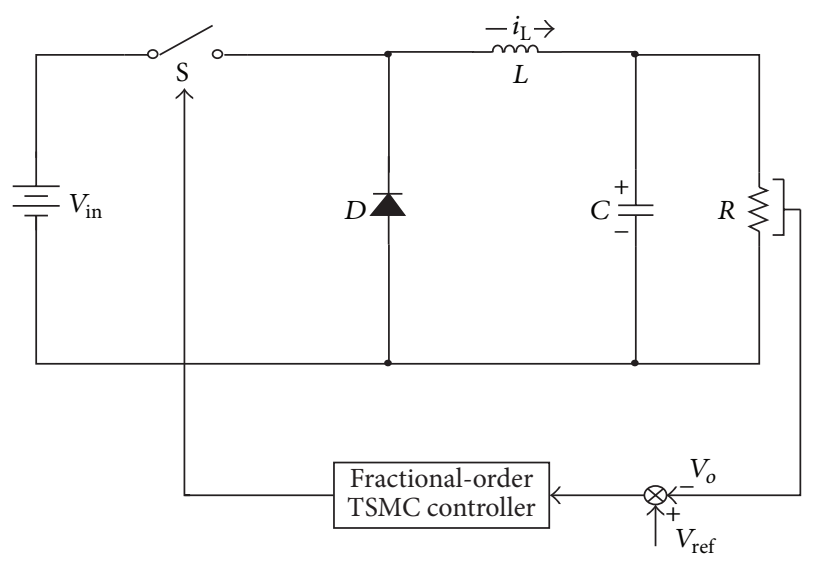

FIGURE 2: Basic structure of an FTMSC Buck converter.

TABLE 1: SMC parameters.

\begin{tabular}{lccc}
\hline Description & $\alpha$ & $\beta$ & $\mu$ \\
\hline SMC & 100 & - & - \\
TSMC & 100 & 0.6 & - \\
FTSMC1 & 100 & 0.6 & 0.9 \\
FTSMC2 & 100 & 0.6 & 0.8 \\
FTSMC3 & 100 & 0.6 & 0.7 \\
FTSMC4 & 100 & 0.6 & 0.6 \\
\hline
\end{tabular}

Therefore, it can be concluded that system trajectories can reach the equilibrium point in finite time. When $\mu=1$, it is obvious that $t_{s}^{\text {TSMC }}$ is equivalent to $t_{s}^{\text {FTSMC }}$. It means that the finite time taken to attain to the equilibrium point of the FTSMC system is the same as the one of the TSMC system as given in (17).

\section{Simulation Results}

The block diagram of the DC/DC Buck converter with the proposed FTSMC method is depicted in Figure 2. In order to show the performance of the proposed methods, the DC/DC Buck converter system has been tested by simulations. Simulations are carried out using Matlab/Simulink. Parameters of Buck converter are given in Abbreviations.

In other literatures, the influences of parameters $\alpha$ and $\beta$ have been studied. This paper focuses on the influence of the order parameter $\mu$ on the control effect. Table 1 shows the parameters of the controller.

As shown in Figure 3, when $\alpha=100$ and $\beta=0.6$, the value range of $\mu$ is between 0 and 1. $\mu-$ Ts curve is not a monotonic function. When the value of $\mu$ is about 0.8 , Ts get the minimum value. The parameter $\mu$ increases the degree of freedom of the original terminal sliding-mode controller. The sliding-mode surface is selected by the original 2 parameters $(\alpha, \beta)$ and becomes the three parameters $(\alpha, \beta, \mu)$.

Figure 4 shows the simulated start-up and transient responses of the output voltage obtained by FTSMC strategies with different $\mu$ values. It is interesting to note that the output

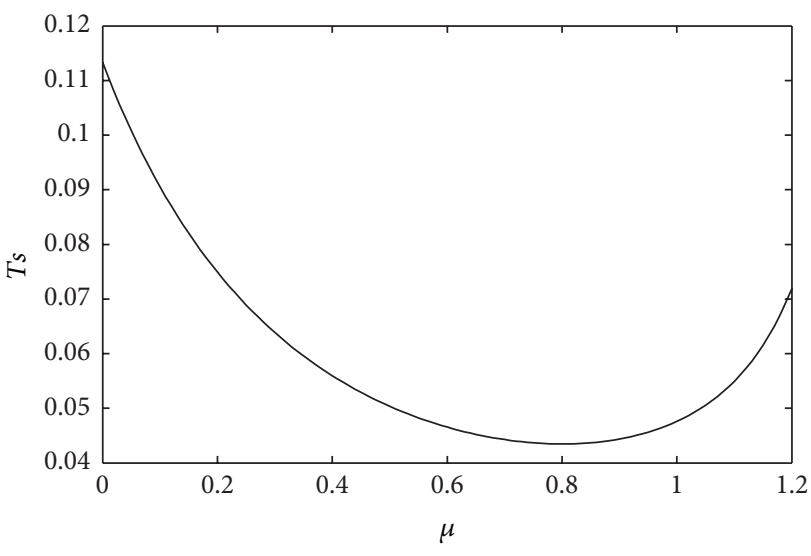

$\alpha=100, \beta=0.6, \mu \in(0,1.2)$

Figure 3: Ts responses due to the changes in $\mu$.

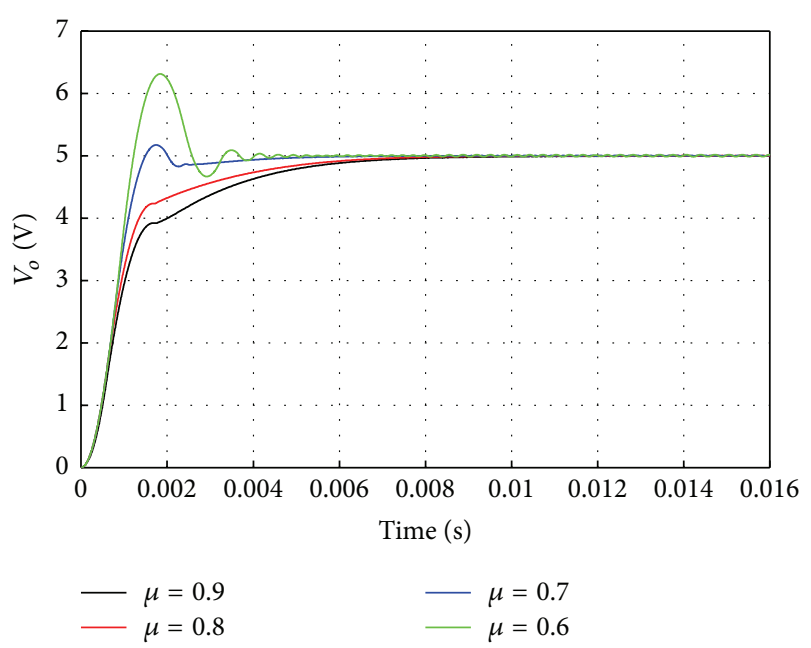

FIGURE 4: Output voltage responses due to the changes in $\mu$.

voltage responses become faster with decreasing the value of $\mu$. Therefore, the value of $\mu$ is chosen to make compromise between start-up and transient responses of the converter. With the decrease of $\mu$, the output voltage response is gradually reduced. When $\mu=0.7$, the overshoot of the system appears. The reason is that, with the decrease of $\mu$, the integral weight is gradually increasing; that is, the cumulative effect on the time scale increases and then affects the system overshoot, which also verifies the influence of $\mu$ on Ts as shown in Figure 3. When $\mu=0.6$, the overshoot of the system exceeds $20 \%$.

In order to compare the control effect of TSMS, FTSMC, and SMC, select $\alpha=100, \beta=0.6$, and $\mu=0.8$. As shown in Figure 5, the response time of the system with FTSMC is less than others. At $t=0.15 \mathrm{~s}$, the load resistance is changed from $2 \Omega$ to $8 \Omega$. Because the load is lighter, the output current will be reduced. Then the output voltage has a short step-up. It can be seen that the output voltage returns faster to $5 \mathrm{~V}$ in FTSMC. 


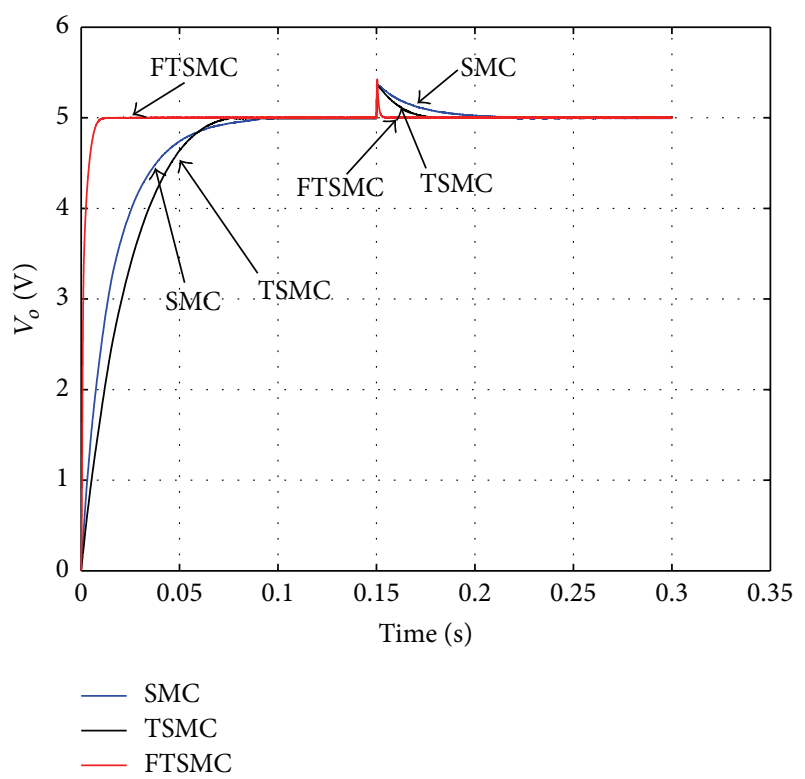

FIGURE 5: Output voltage responses due to the step changes in $R$ from $2 \Omega$ to $8 \Omega$ at $t=0.15 \mathrm{~s}$.

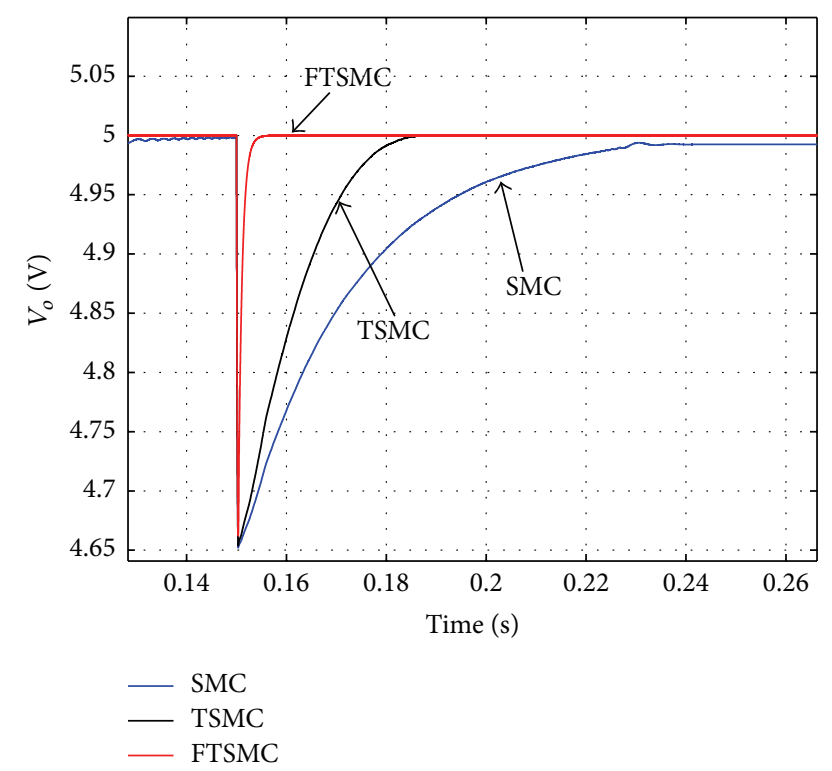

FIgURE 6: Output voltage responses due to the step changes in $R$ from $8 \Omega$ to $2 \Omega$ at $t=0.15 \mathrm{~s}$.

Figure 6 shows the output voltage $V_{o}$ responses due to the step changes in $R$ from $8 \Omega$ to $2 \Omega$. We can find the same results as above. The control effect of FTSMC is better.

It is obvious that the FTSMC method acts faster than others in correcting the output voltage when a step change takes place in $V_{\text {in }}$. On the other hand, as shown in Figure 7, it is clear that SMC and TSMC result in a slight steady-state error. By contrast, the FTSMC method has the lesser steadystate error.

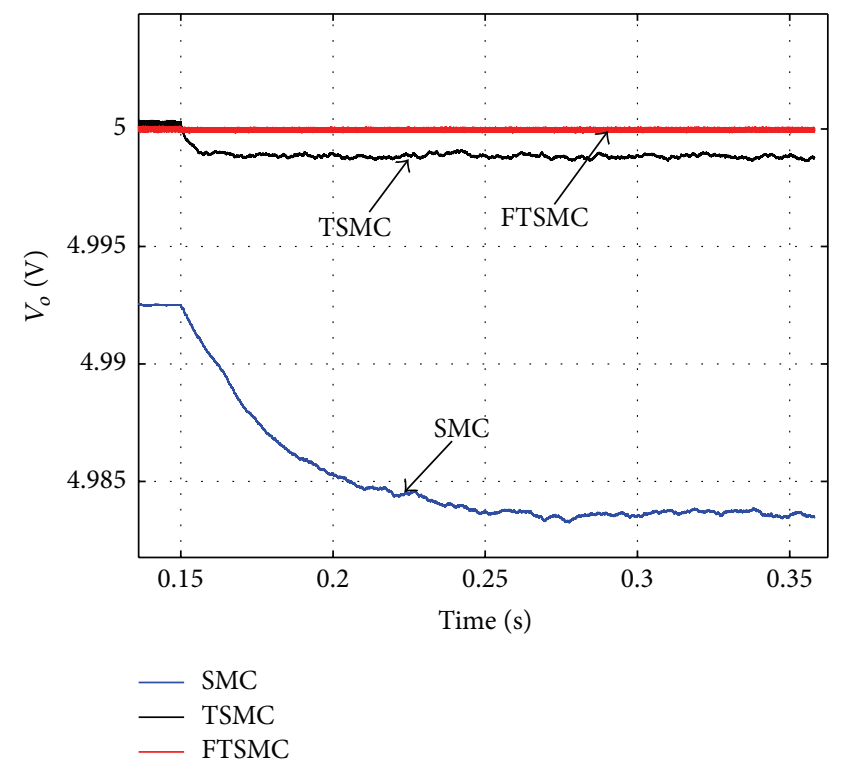

FIGURE 7: Output voltage responses due to the step changes in $V_{\text {in }}$ from $10 \mathrm{~V}$ to $9 \mathrm{~V}$ at $t=0.15 \mathrm{~s}$.

\section{Conclusions}

This paper presents the fractional-order terminal slidingmode control (FTSMC) which has a new fractional-order surface and assures the finite time convergence of the output voltage error to the equilibrium point during the load changes. It introduces a novel tuning parameter, the order $\mu$, which allows the adjustment of the system output accordingly. Through mathematical analysis, we obtain the finite time of fractional-order terminal sliding-mode control. It is shown that when the order $\mu$ of FTSMC is one, the finite time taken to attain to the equilibrium point of the FTSMC system is the same as the one of the TSMC system. In other words, it means that TMSC is a special case of FTMSC. In addition, simulation results for a Buck converter are given to show the good performance of the FTSMC in comparison with the use of the traditional TSMC and SMC. The novel fractional terminal sliding-mode control exhibits considerable improvement in terms of a faster output voltage response during load changes.

\section{Abbreviations}

Specifications of Buck Converter

$V_{\text {in }}: \quad$ Input voltage $(10 \mathrm{~V})$

C: $\quad$ Capacitance $(1000 \mu \mathrm{F})$

$L: \quad$ Inductance $(1 \mathrm{mH})$

$R_{\text {min }}:$ Minimum load resistance $(2 \Omega)$

$R_{\max }$ : Maximum load resistance $(8 \Omega)$

$V_{o}$ : Desired output voltage $(5 \mathrm{~V})$.

\section{Competing Interests}

The authors declare that they have no competing interests. 


\section{Acknowledgments}

This work was supported by the National Natural Science Foundation of China (Grant nos. 51507134 and 51279161), Scientific Research Program Funded by Shaanxi Provincial Education Department (Program no. 15JK1537), and Doctoral Scientific Research Foundation of Xian Polytechnic University (Grant no. BS15025).

\section{References}

[1] K. B. Oldham and J. Spanier, The Fractional Calculus, Academic Press, New York, NY, USA, 1974.

[2] B. B. Xu, D. Y. Chen, H. Zhang, and F. Wang, "The modeling of the fractional-order shafting system for a water jet mixedflow pump during the startup process," Communications in Nonlinear Science and Numerical Simulation, vol. 29, no. 1-3, pp. 12-24, 2015.

[3] N.-N. Yang, C.-X. Liu, and C.-J. Wu, "Modeling and dynamics analysis of the fractional-order Buck-boost converter in continuous conduction mode," Chinese Physics B, vol. 21, no. 8, 2012.

[4] N. N. Yang, C. J. Wu, R. Jia, and C. Liu, "Modeling and characteristics analysis for a buck-boost converter in pseudocontinuous conduction mode based on fractional calculus," Mathematical Problems in Engineering, vol. 2016, Article ID 6835910, 11 pages, 2016.

[5] C. Wu, G. Si, Y. Zhang, and N. Yang, "The fractional-order statespace averaging modeling of the Buck-Boost DC/DC converter in discontinuous conduction mode and the performance analysis," Nonlinear Dynamics, vol. 79, no. 1, pp. 689-703, 2015.

[6] N.-N. Yang, C.-X. Liu, and C.-J. Wu, "A hyperchaotic system stabilization via inverse optimal control and experimental research," Chinese Physics B, vol. 19, no. 10, Article ID 100502, 2010.

[7] I. Podlubny, Fractional Differential Equations, vol. 198, Academic Press, New York, NY, USA, 1999.

[8] D. Y. Chen, R. F. Zhang, X. Z. Liu, and X. Ma, "Fractional order Lyapunov stability theorem and its applications in synchronization of complex dynamical networks," Communications in Nonlinear Science and Numerical Simulation, vol. 19, no. 12, pp. 4105-4121, 2014.

[9] A. S. Hegazi, E. Ahmed, and A. E. Matouk, "On chaos control and synchronization of the commensurate fractional order Liu system," Communications in Nonlinear Science and Numerical Simulation, vol. 18, no. 5, pp. 1193-1202, 2013.

[10] C.-J. Wu, Y.-B. Zhang, and N.-N. Yang, "The synchronization of a fractional order hyperchaotic system based on passive control," Chinese Physics B, vol. 20, no. 6, Article ID 060505, 2011.

[11] D. Y. Chen, R. F. Zhang, J. C. Sprott, and X. Ma, "Synchronization between integer-order chaotic systems and a class of fractional-order chaotic system based on fuzzy sliding mode control," Nonlinear Dynamics, vol. 70, no. 2, pp. 1549-1561, 2012.

[12] A. Oustaloup, X. Moreau, and M. Nouillant, "The CRONE suspension," Control Engineering Practice, vol. 4, no. 8, pp. 11011108, 1996.

[13] I. Podlubny, "Fractional-order systems and PI $\lambda \mathrm{D} \mu$-controllers," IEEE Transactions on Automatic Control, vol. 44, no. 1, pp. 208214, 1999.

[14] X. Wang, X. Zhang, and C. Ma, "Modified projective synchronization of fractional-order chaotic systems via active sliding mode control," Nonlinear Dynamics, vol. 69, no. 1-2, pp. 511-517, 2012.

[15] N. N. Yang and C. X. Liu, "A novel fractional-order hyperchaotic system stabilization via fractional sliding-mode control," Nonlinear Dynamics, vol. 74, no. 3, pp. 721-732, 2013.

[16] D. Y. Chen, R. F. Zhang, X. Y. Ma, and S. Liu, "Chaotic synchronization and anti-synchronization for a novel class of multiple chaotic systems via a sliding mode control scheme," Nonlinear Dynamics, vol. 69, no. 1-2, pp. 35-55, 2012.

[17] C. Tricaud and Y. Q. Chen, "An approximate method for numerically solving fractional order optimal control problems of general form," Computers and Mathematics with Applications, vol. 59, no. 5, pp. 1644-1655, 2010.

[18] S. Ladaci and A. Charef, "On fractional adaptive control," Nonlinear Dynamics, vol. 43, no. 4, pp. 365-378, 2006.

[19] C. J. Wu, G. Q. Si, Y. B. Zhang, and N. Yang, "Adaptive inverse optimal control of a novel fractional-order four-wing hyperchaotic system with uncertain parameter and circuitry implementation," Mathematical Problems in Engineering, vol. 2015, Article ID 741307, 15 pages, 2015.

[20] S.-C. Tan, Y.-M. Lai, and C. K. Tse, Sliding Mode Control of Switching Power Converters: Techniques and Implementation, CRC Press, Boca Raton, Fla, USA, 2011.

[21] M. J. Jafarian and J. Nazarzadeh, "Time-optimal sliding-mode control for multi-quadrant buck converters," IET Power Electronics, vol. 4, no. 1, pp. 143-150, 2011.

[22] R. Ramos, D. Biel, E. Fossas, and R. Griño, "Sliding mode controlled multiphase buck converter with interleaving and current equalization," Control Engineering Practice, vol. 21, no. 5, pp. 737-746, 2013.

[23] K. K.-S. Leung and H. S.-H. Chung, "A comparative study of boundary control with first- and second-order switching surfaces for buck converters operating in DCM," IEEE Transactions on Power Electronics, vol. 22, no. 4, pp. 1196-1209, 2007.

[24] B. M. Vinagre and A. J. Calderon, "On fractional sliding mode control," in Proceedings of the 7th Portuguese Conference on Automatic Control, Lisbon, Portugal, September 2006.

[25] H. Delavari, R. Ghaderi, A. Ranjbar, and S. Momani, "Fuzzy fractional order sliding mode controller for nonlinear systems," Communications in Nonlinear Science and Numerical Simulation, vol. 15, no. 4, pp. 963-978, 2010.

[26] A. J. Calderón, B. M. Vinagre, and V. Feliu, "Fractional order control strategies for power electronic buck converters," Signal Processing, vol. 86, no. 10, pp. 2803-2819, 2006.

[27] S. H. Hosseinnia, I. Tejado, B. M. Vinagre, and D. Sierociuk, "Boolean-based fractional order SMC for switching systems: application to a DC-DC buck converter," Signal, Image and Video Processing, vol. 6, no. 3, pp. 445-451, 2012. 


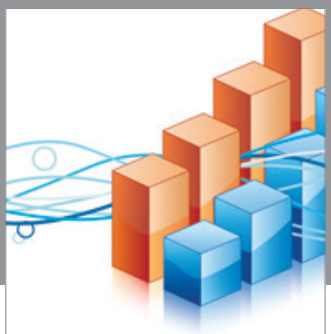

Advances in

Operations Research

vatem alat4

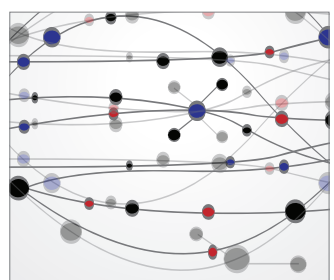

\section{The Scientific} World Journal
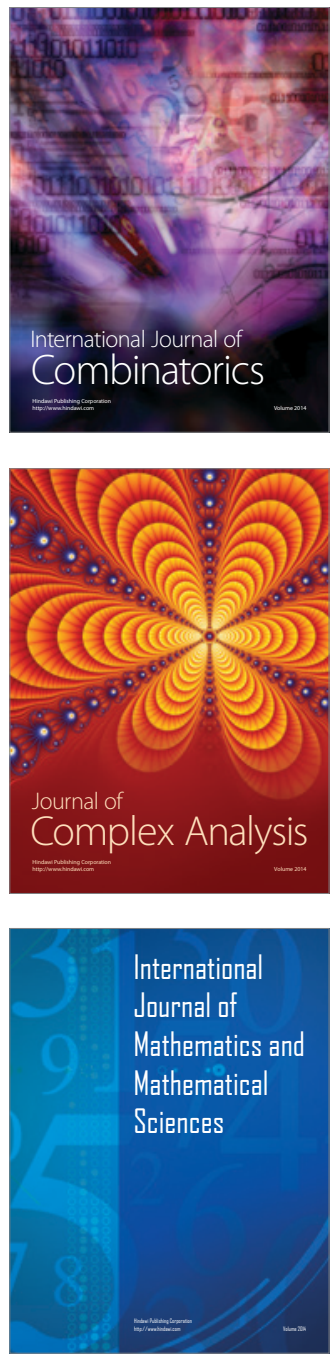
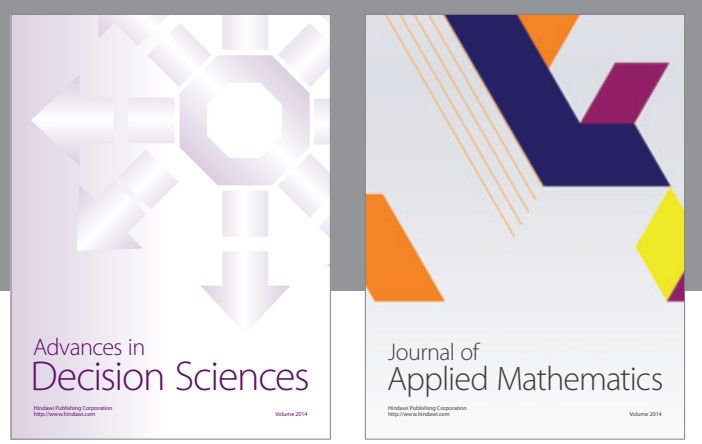

Algebra

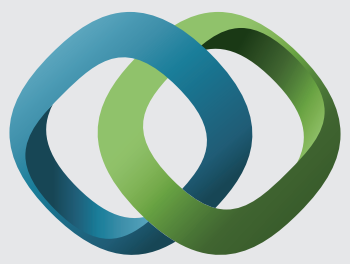

\section{Hindawi}

Submit your manuscripts at

http://www.hindawi.com
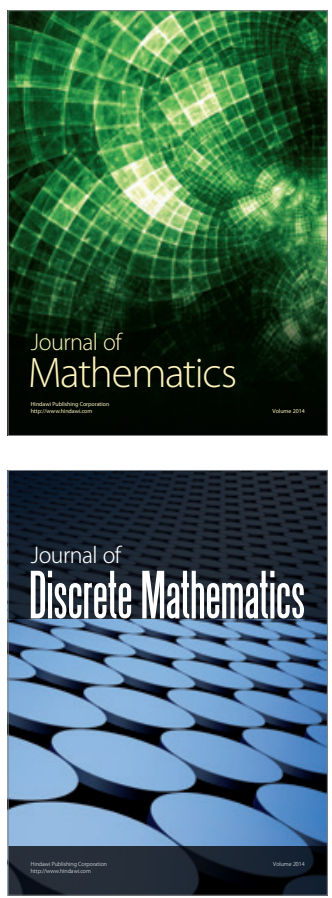

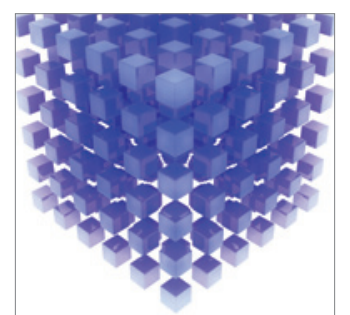

Mathematical Problems in Engineering
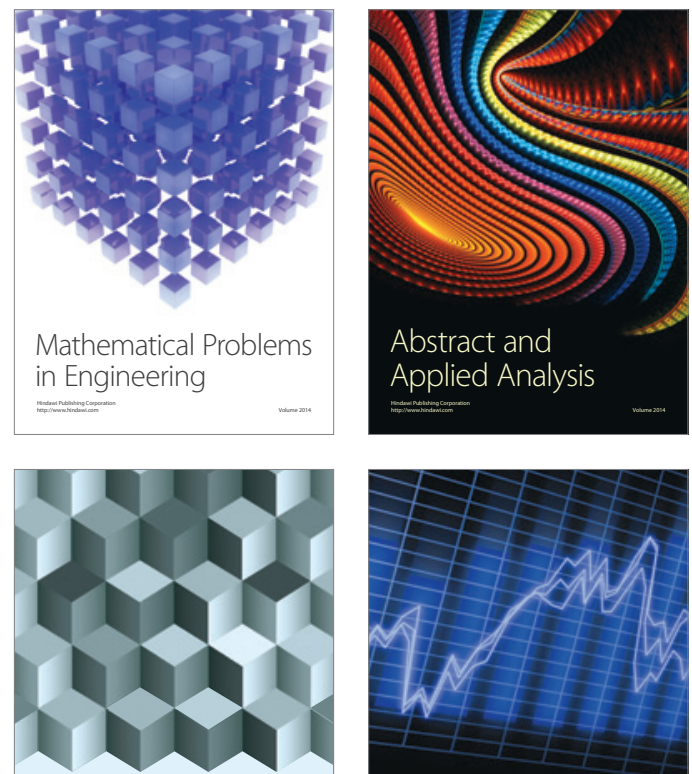

Journal of

Function Spaces

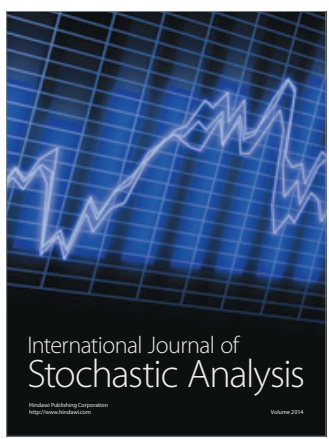

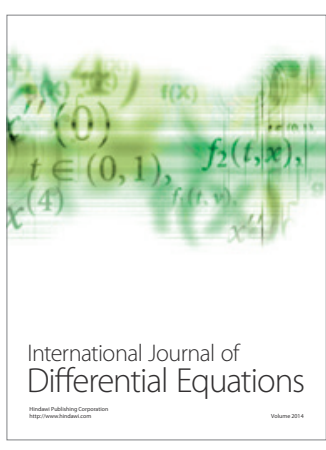
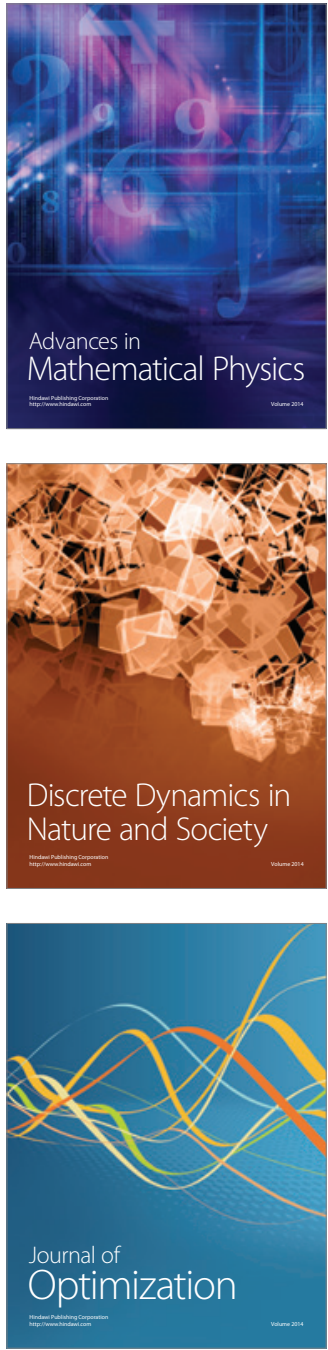